\title{
Optimal control with nonadiabatic molecular dynamics: Application to the Coulomb explosion of sodium clusters
}

\author{
Adrián Gómez Pueyo* and Jorge A. Budagosky M. \\ Institute for Biocomputation and Physics of Complex Systems, University of Zaragoza, Calle Mariano Esquillor, 50018 Zaragoza, Spain \\ Alberto Castro \\ Institute for Biocomputation and Physics of Complex Systems, University of Zaragoza, Calle Mariano Esquillor, 50018 Zaragoza, Spain \\ and ARAID Foundation, Calle María Luna, 50018 Zaragoza, Spain
}

(Received 27 June 2016; revised manuscript received 11 November 2016; published 27 December 2016)

\begin{abstract}
We present an implementation of optimal control theory for the first-principles nonadiabatic Ehrenfest molecular dynamics model, which describes a condensed matter system by considering classical point-particle nuclei, and quantum electrons, handled in our case with time-dependent density-functional theory. The scheme is demonstrated by optimizing the Coulomb explosion of small sodium clusters: the algorithm is set to find the optimal femtosecond laser pulses that disintegrate the clusters, for a given total duration, fluence, and cutoff frequency. We describe the numerical details and difficulties of the method.
\end{abstract}

DOI: 10.1103/PhysRevA.94.063421

\section{INTRODUCTION}

Thanks to the rapid development of laser technology [1], we have nowadays access to high-intensity, short-duration pulse sources. This availability has led to the birth of femtosecond science [2] in the last decades of the 20th century, and of attosecond science [3] in the new century. It has become possible to study, in real time, the evolution of ions and electrons as they evolve in the influence of short and intense laser pulses.

Theoretically, molecular dynamics (MD) [4] studies the processes that undergo condensed matter systems, in and out of equilibrium, through simulations. For more than a few particles, it is impossible to achieve a full exact quantum description of the problem. Often, the nuclei are considered classical in an effort to alleviate the difficulties. In fact, the name "molecular dynamics" is traditionally reserved for these models in which the nuclear degrees of freedom are classical. Unfortunately, the remaining electronic quantum problem in a nonequilibrium situation is still very challenging [5]. Moreover, the electron-nuclear coupling is in general nonadiabatic: in the adiabatic approximation, the electronic system is fixed to the ground state corresponding to the instantaneous nuclear configuration, which is obviously unsuitable for the highly excited situation that high-intensity sudden laser pulses lead to. For a good description, one must use a first-principles theory for the nonequilibrium many-electron quantum dynamics, nonadiabatically coupled to the classic ionic movement through some form of MD.

One such scheme is the Ehrenfest molecular dynamics (EMD) in combination with time-dependent densityfunctional theory (TDDFT) [6,7]. EMD involves two approximations: first, one separates the electronic and nuclear parts of the full wave function, arriving at a "time-dependent self-consistent model" [8]; second, the short wave asymptotics idea [9-11] is used to take the classical limit for the nuclear equations (see Ref. [12] for details on the justification of

\footnotetext{
*agomez@bifi.es
}

these steps). Then, one still needs to treat the many-electron system by making use of some electronic structure theory, such as TDDFT. Ehrenfest MD based on TDDFT was first attempted by Theilhaber [13] for external free-field problems, and its applicability for laser-matter interaction was proved with various examples [14-19].

The versatility of laser sources soon allowed the possibility of controlling quantum dynamics, a step beyond its mere use for spectroscopy. The field of coherent or quantum control [20-30] was thus born. A corresponding theoretical framework had to be attached to the experimental advances. This is optimal control theory (OCT), the mathematical branch that studies the "inverse" question in the study of dynamical systems: given a set of equations of motion that depend on a set of parameters, OCT studies the methods to find the parameters that optimize the system evolution. The first theoretical calculations of OCT for quantum processes (QOCT) were reported in the 1980s [31-34], and the field quickly developed in the following decades $[30,35]$.

OCT has been demonstrated, for example, on the quantum equations for the evolution of nuclear wave packets, with the aim of controlling nuclear reactions. It was also combined with TDDFT [36], which opened the road to the direct control of electronic systems. An obvious step forward is the combination of OCT with an ab initio nonadiabatic MD scheme, which implies a control theory for a mixed quantumclassical system. OCT for classical or mixed quantum-classical systems was already explored, for example, in Refs. [37,38]. From a first-principles perspective, the EMD-TDDFT scheme is a good candidate to attempt its combination with OCT, due to its simplicity. In Ref. [39] the essential equations of an OCT for the EMD-TDDFT scheme were presented, while Ref. [40] demonstrated its numerical feasibility for some simple two-electron molecules $\left(\mathrm{H}_{2}\right.$ and $\left.\mathrm{H}_{3}{ }^{+}\right)$. We have further developed this numerical implementation, to allow for larger and more complicated systems, and it is the purpose of this article to describe it in detail and show how it can be employed for some larger systems. In particular, we have attempted the optimization of the Coulomb explosion of small sodium clusters: the algorithm is set to 
find the optimal femtosecond laser pulses that disintegrate the clusters for a given total duration, fluence, and cutoff frequency.

We have chosen the Coulomb explosion of sodium clusters because (1) it is a far-from-equilibrium violent process [41] that requires a nonperturbative scheme such as the one used here; (2) the high-intensity irradiation of simple metal clusters was successfully studied with TDDFT in the past $[42,43]$; (3) the Coulomb explosion of these systems was also already treated with EMD-TDDFT [15,44,45], and it was shown how there is an interesting interplay between the laser pulse, the electrons, and the ionic motion.

Indeed, in principle the massive ionization that is responsible for the Coulomb explosion may be achieved by tuning the laser frequency to the large surface plasmon using the resonance-enhanced ionization mechanism. One could then think that the explosion optimization problem can be simply solved by making use of correctly tuned monochromatic pulses. However, the clusters change during the pulse action in two different ways that complicate the picture: (1) as ionization increases, the main resonance blueshifts, and (2) as the ions start to move and separate from each other, the main resonance redshifts. It is therefore impossible to predict a priori how to "correct" a simple, initially resonant, monochromatic pulse with other components in order to fully optimize the explosion process. This makes it a task suited for OCT.

In Sec. II we present the theoretical framework which is at the base of this work. In Sec. III we present the results obtained for the Coulomb explosion of $\mathrm{Na}_{2}, \mathrm{Na}_{4}$, and $\mathrm{Na}_{8}$ clusters. In Sec. IV we discuss the implication of the results found. Finally, in the Appendix we detail the key aspects of the computational implementation. Atomic units are used hereafter unless stated otherwise.

\section{OCT FOR THE EMD-TDDFT MODEL}

The details of the theory were given in Ref. [39]; we only outline here the key equations. An Ehrenfest system is a hybrid quantum-classical system whose state is specified by a set of classical conjugated position and momenta variables $\{R, P\}=\left\{\vec{R}_{\alpha}, \vec{P}_{\alpha}\right\}_{\alpha=1}^{K}$ (where $\alpha$ runs over the $K$ nuclei of our system, each with mass $M_{\alpha}$ and charge $z_{\alpha}$ ) and the many-body wave function $\Psi$ of the $N$ electrons of our system. The forces that determine the nuclear movement are given by

$$
\begin{aligned}
\vec{F}_{\alpha}[R(t), \Psi(t), u, t]= & -\vec{\nabla}_{\alpha} W^{n n}(R(t))+z_{\alpha} \varepsilon(u, t) \vec{\pi} \\
& -\left\langle\Psi(t)\left|\vec{\nabla}_{\alpha} \hat{H}[R(t), u, t]\right| \Psi(t)\right\rangle,
\end{aligned}
$$

where the nucleus-nucleus interaction $W^{n n}$ takes the usual Coulomb form

$$
W^{n n}(R)=\sum_{\beta<\gamma} \frac{z_{\beta} z_{\gamma}}{\left|\vec{R}_{\beta}-\vec{R}_{\gamma}\right|} .
$$

The time-dependent function $\varepsilon(u, t)$ is the amplitude of the laser pulse, which is polarized along the unit vector $\vec{\pi}$. The magnetic component is ignored, as we assume the dipole approximation and the length gauge. $u=\left\{u_{1}, u_{2}, \ldots, u_{M}\right\}$ is the set of parameters that can be controlled and give the precise shape to the laser. Finally, the last term in Eq. (1) contains the electronic Hamiltonian, given by

$$
\begin{aligned}
\hat{H}[R, u, t]= & \sum_{i=1}^{N} \frac{\hat{\vec{p}}_{i}^{2}}{2}+\sum_{i<j} \frac{1}{\left|\hat{\vec{r}}_{i}-\hat{\vec{r}}_{j}\right|}+\varepsilon(u, t) \sum_{i=1}^{N} \hat{\vec{r}}_{i} \cdot \vec{\pi} \\
& +\sum_{i=1}^{N} \sum_{\beta}^{K} v^{\beta}\left(\left|\hat{\vec{r}}_{i}-\vec{R}_{\beta}(t)\right|\right),
\end{aligned}
$$

where $\hat{\vec{r}}_{i}$ and $\hat{\vec{p}}_{i}$ are the position and momentum operators, respectively, for the $i$ th electron. The electron-nucleus interaction in the last term is usually the Coulomb form $v^{\beta}(r)=-\frac{z_{\beta}}{r}$, but in practice we use pseudopotentials [46], which may $\stackrel{r}{\text { be }}$ nonlocal operators.

Rather than using the many-electron wave function, we want to use the TDDFT Kohn-Sham (KS) formalism to substitute the real electronic system with an equivalent one where the electrons do not interact with each other. This set of electrons can be described using a single Slater determinant formed by $N$ spin orbitals. The time-dependent electronic densities of both systems are identical by construction. It is given, in terms of the KS orbitals, by

$$
n_{t}(\vec{r}) \equiv n(\vec{r}, t)=\sum_{m=1}^{N / 2} 2\left|\varphi_{m}(\vec{r}, t)\right|^{2}
$$

This assumes a spin-restricted situation in which we have an even number of electrons, no magnetic fields, and no spin-orbit coupling. The system evolves in a spin singlet and the spin orbitals are paired, each pair sharing the same orbital part $\left(\varphi_{m}\right)$ at all times. The examples presented below assume this configuration.

The KS orbitals evolve according to the KS equations, a set of nonlinear Schrödinger-like equations:

$$
i \frac{d}{d t}\left|\varphi_{i}(t)\right\rangle=\hat{H}_{\mathrm{KS}}\left[R(t), n_{t}, u, t\right]\left|\varphi_{i}(t)\right\rangle \quad(i=1, \ldots, N / 2) .
$$

The KS Hamiltonian $\hat{H}_{\mathrm{KS}}$ is defined by

$$
\begin{aligned}
\hat{H}_{\mathrm{KS}}\left[R(t), n_{t}, u, t\right]= & \frac{1}{2} \hat{\vec{p}}^{2}+\sum_{\beta} \hat{v}^{\beta}\left(\left|\hat{\vec{r}}-\vec{R}_{\beta}(t)\right|\right)+\varepsilon(u, t) \hat{\vec{r}} \cdot \vec{\pi} \\
& +\int d^{3} r^{\prime} \frac{n_{t}\left(\vec{r}^{\prime}\right)}{\left|\vec{r}-\vec{r}^{\prime}\right|}+v_{\mathrm{xc}}\left[n_{t}\right](\vec{r}) .
\end{aligned}
$$

The next-to-last term is the Hartree potential, and the last one is the exchange-correlation potential. We assume an adiabatic approximation for this last term, i.e., at each time $t$ it only depends on the density at that time $n_{t}$. We have used, in particular, the adiabatic local density approximation (ALDA), which is the first-step approximation to the intricate problem of the exchange and correlation functional. However, the details of the methodology presented here do not depend on the approximation used, and we have preferred to use this generic choice because it is sufficient to draw qualitative conclusions and was successfully used in the past for similar simulations [44]. 
The force on each nucleus given in Eq. (1) can be expressed as a function of the time-dependent density, or alternatively, in terms of the KS orbitals $\varphi \equiv\left\{\varphi_{m}\right\}_{m=1}^{N / 2}$ :

$$
\begin{aligned}
\vec{F}_{\alpha}[R(t), \varphi(t), u, t]= & -\vec{\nabla}_{\alpha} W^{n n}(R(t))+z_{\alpha} \varepsilon(u, t) \vec{\pi} \\
& -\sum_{m=1}^{N / 2} 2\left\langle\varphi_{m}(t)\left|\vec{\nabla}_{\alpha} v^{\alpha}\left(\left|\hat{\vec{r}}-\hat{\vec{R}}_{\alpha}(t)\right|\right)\right| \varphi_{m}(t)\right\rangle .
\end{aligned}
$$

This fact permits us to avoid the full many-electron wave function completely and is the basis of the EMD-TDDFT method. The full set of equations of motion is given by Eqs. (5) plus the usual classical Newton equations for the nuclear movement:

$$
\begin{gathered}
\dot{\vec{R}}_{\alpha}(t)=\frac{1}{M_{\alpha}} \vec{P}_{\alpha}, \\
\dot{\vec{P}}_{\alpha}(t)=\vec{F}_{\alpha}[R(t), \varphi(t), u, t] .
\end{gathered}
$$

Our purpose now is to look for the set of parameters $u$ that optimize the behavior of the system with respect to a physical goal, e.g., the population of some excited electronic state, the cleavage of a particular bond, or, as it is the case of the examples shown below, the Coulomb explosion of a cluster. This needs to be formulated as the maximization of a target functional that depends on the system variables:

$$
\mathcal{F}=\mathcal{F}\left[R(T), P(T), n_{T}, u\right],
$$

where the time $T$ is the final time of the propagation interval $[0, T]$. This definition depends only on the state of the system at the end of the propagation interval (one could also formulate OCT for an evolution-dependent target).

Each set of parameters $u$ determines the evolution of the system, $u \rightarrow R[u], P[u], \varphi[u]$, so that the problem is reduced to the maximization of a function:

$$
G(u)=\mathcal{F}\left[R(T), P(T), n_{T}, u\right] .
$$

The algorithms for the maximization of functions are superior if they can make use of the gradient of the function. One of the main results of OCT is the derivation of an expression for the gradient; for the MD model that we are discussing, the expression is [39]

$$
\begin{gathered}
\frac{\partial G}{\partial u_{k}}=\int_{0}^{T} d t \frac{\partial \varepsilon}{\partial u_{k}}(u, t) g(t), \\
g(t)=-\sum_{\beta} z_{\beta} \tilde{\vec{R}}_{\beta}(t) \cdot \vec{\pi}+2 \operatorname{Im} \sum_{m=1}^{N / 2}\left\langle\chi_{m}(t)|\hat{\vec{r}} \cdot \vec{\pi}| \varphi_{m}(t)\right\rangle .
\end{gathered}
$$

In this expression, there are new objects: new position variables $\tilde{\vec{R}}_{\beta}$ and one-particle orbitals $\left\{\chi_{m}\right\}_{m=1}^{N / 2}$. Together with new momenta variables $\tilde{\vec{P}}_{\beta}$, they form the sometimes-called costate, an auxiliary mixed quantum and classical system whose evolution is given by the following equations of motion:

$$
\begin{gathered}
\dot{\overrightarrow{\vec{R}}}_{\alpha}(t)=\frac{1}{M_{\alpha}} \tilde{\vec{P}}_{\alpha}(t) \\
\dot{\overrightarrow{\vec{P}}}_{\alpha}(t)=\vec{\nabla}_{\alpha} \sum_{\beta} \tilde{\vec{R}}_{\beta}(t) \cdot \vec{F}_{\beta}[R(t), \varphi(t), u, t] \\
+2 \operatorname{Re} i \sum_{m=1}^{N / 2}\left\langle\chi_{m}(t)\left|\vec{\nabla}_{\alpha} \hat{H}_{\mathrm{KS}}\left[R(t), n_{t}, u, t\right]\right| \varphi_{m}\right\rangle \\
\left|\dot{\chi}_{m}(t)\right\rangle=-i \hat{H}_{\mathrm{KS}}[R(t), u, t]\left|\varphi_{m}\right\rangle-i \sum_{n=1}^{N / 2} \hat{K}_{m n}[\varphi(t)]\left|\chi_{n}(t)\right\rangle \\
\\
-2 \sum_{\beta} \tilde{\vec{R}}_{\beta} \cdot \vec{\nabla}_{\beta} \hat{v}^{\beta}\left(\left|\hat{\vec{r}}-\vec{R}_{\beta}(t)\right|\right)\left|\varphi_{m}(t)\right\rangle
\end{gathered}
$$

The evolution equation for the costate orbitals $\chi_{m}$ contains a new, non-Hermitian term $\hat{K}_{m n}$, defined by

$$
\begin{aligned}
\left\langle\vec{r}\left|\hat{K}_{m n}[\varphi(t)]\right| \chi_{m}(t)\right\rangle= & -4 i \varphi_{m}(\vec{r}, t) \operatorname{Im} \int d^{3} r^{\prime} \chi_{n}(\vec{r}, t) f_{\mathrm{Hxc}} \\
& \times\left[n_{t}\right]\left(\vec{r}, \vec{r}^{\prime}\right) \varphi_{n}(\vec{r}, t),
\end{aligned}
$$

where the kernel $f_{\mathrm{Hxc}}$ is the functional derivative of the Hartree and exchange and correlation potential functionals:

$$
f_{\mathrm{Hxc}}[n]\left(\vec{r}, \vec{r}^{\prime}\right)=\frac{1}{\left|\vec{r}-\vec{r}^{\prime}\right|}+\frac{\delta v_{\mathrm{xc}}[n](\vec{r})}{\delta n\left(\vec{r}^{\prime}\right)} .
$$

Of course, the equations of motion for the costate need boundary conditions:

$$
\begin{gathered}
\tilde{\vec{R}}_{\alpha}(T)=-\frac{\partial}{\partial \vec{P}_{\alpha}} \mathcal{F}(R(T), P(T)), \\
\tilde{\vec{P}}_{\alpha}(T)=\frac{\partial}{\partial \vec{R}_{\alpha}} \mathcal{F}(R(T), P(T)), \\
\left\langle\vec{r} \mid \chi_{m}(T)\right\rangle=\frac{\delta \mathcal{F}}{\delta \varphi_{m}^{*}(\vec{r}, T)} .
\end{gathered}
$$

These are final value conditions, and therefore the computation of the components of the $G$ gradient implies a forward propagation of the equations of motion of our system and then a backwards propagation of the equations of motion for the costate [Eqs. (14), (15), and (16)], together with the previous final value conditions.

We have implemented these equations in the OCTOPUS code $[47,48]$. The orbitals, densities, potentials, etc. are represented in real space in a regular rectangular grid (for the calculations shown below, the grid spacing was set to 0.8 a.u.) and the system is contained in a simulation box $V$ (that was chosen to be spherical for $\mathrm{Na}_{2}$ and $\mathrm{Na}_{8}$, and cylindrical for $\mathrm{Na}_{4}$ ), with zero boundary conditions in the edges. During the simulations, we add an imaginary absorbing potential in the borders of the simulation box to simulate the ionization of the electrons [see Eq. (A9) in the Appendix]. Essentially, the problem consists of computing the maximum of the function $G$ defined above [Eq. (11)], which we find with the help of the Broyden-Fletcher-Goldfarb-Shanno (BFGS) algorithm $[49,50]$. The gradient necessitated by that algorithm is obtained via the forward and backwards propagations mentioned above, propagations that are done with the standard explicit fourthorder Runge-Kutta scheme. 
More details about the numerics are given in the Appendix, with particular attention to the the key computational challenges posed by the integration of OCT with the EMD-TDDFT method.

\section{APPLICATIONS}

We have applied the methodology described above to the following problem: find the optimal laser pulses that Coulomb-explode the $\mathrm{Na}_{2}, \mathrm{Na}_{4}$, and $\mathrm{Na}_{8}$ clusters for a given total duration, fluence, and cutoff frequency. The first step is to fix an initial ground-state ionic configuration. We have started from reference geometries found in the literature [51-53] and have further relaxed them with our own code in order to start the calculations with exactly zero force. As expected, the modified geometries are similar to the reference ones, although we did find the known tendency of the local density approximation (LDA) to underestimate bond lengths.

Next, we define a "reference" pulse in the following form:

$$
\varepsilon(t)=A e^{-\left(t-t_{0}\right)^{2} / 2 \eta^{2}} \sin \left(\omega_{0} t\right) .
$$

The frequency $\omega_{0}$ is set to $800 \mathrm{~nm}$, the constant $t_{0}$ that determines the position of the peak is set to half of the total pulse duration, and the constant $\eta$ that determines the width is set to $0.9 \tau_{0}$, where $\tau_{0}=2 \pi / \omega_{0}$ is the period of the laser pulse. The search space is then defined by fixing the total fluence to that of this reference pulse, which can be tuned through the amplitude constant $A$. The total duration $T$ of the pulses allowed in the search space is set to 6,12 , or 24 periods $\tau_{0}$ of the reference frequency of $800 \mathrm{~nm}(\sim 16,32$, or $64 \mathrm{fs})$, depending on the case. We further impose a cutoff frequency for the control function $\omega_{\text {cutoff }}=0.5 \mathrm{Ha}$, and the search space is then parametrized as discussed in the Appendix, point 6 . The optimization process is started with an initial random shape, and in order to assure that the parameter space was adequately explored, we did several runs for each case.

We then performed OCT runs in three different manners:

(1) Using a target defined in terms of the classical momenta of the nuclei, i.e.,

$$
\mathcal{F}[P(T)]=\sum_{i<j}\left|\vec{P}_{i}(T)-\vec{P}_{j}(T)\right|^{2},
$$

where $\vec{P}_{i}(T)$ is the momentum of the $i$ th nucleus at the end of the propagation. The rationale behind this definition is clear: at the end of the pulse, the ions in the cluster are required to have the maximum possible opposing momenta.

(2) Using as a target an estimation for the ionization, i.e.,

$$
\mathcal{F}\left[n_{T}\right]=-\int_{V} n_{T}(\vec{r}) d^{3} r,
$$

where $n_{T}(\vec{r})$ is the electronic density at the end of the pulse action, $t=T$. This amounts to the maximization of the electronic escape of the system, which is what triggers the Coulomb explosion. First, we performed optimizations with this target but fixing the nuclei to their equilibrium position during the action of the pulse. This means that we are not, in this second case, using the mixed quantum and classical OCT scheme stated in Sec. II but only the QOCT for TDDFT described in Ref. [36].

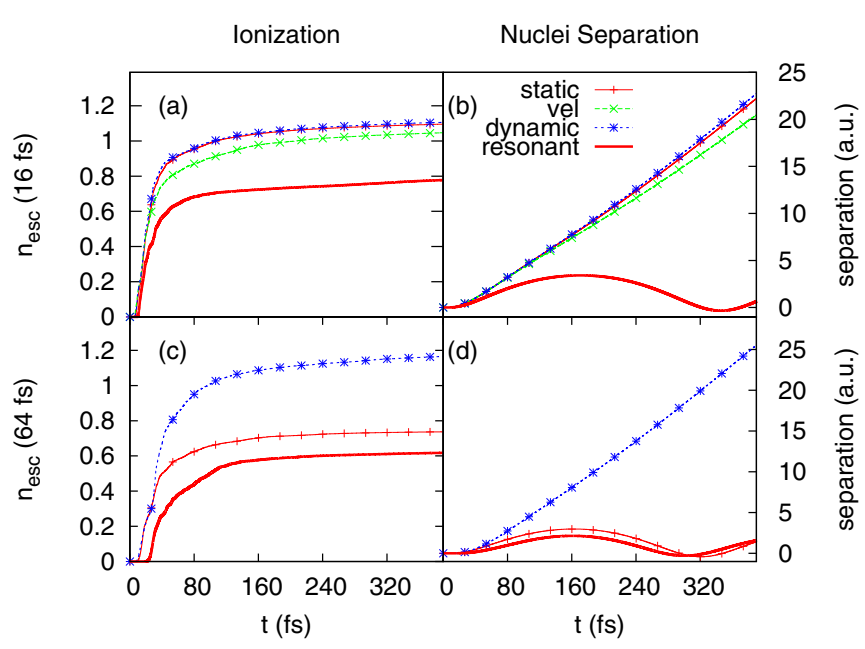

FIG. 1. Left panels $(\mathrm{a}, \mathrm{c})$ : Electrons escaped from the $\mathrm{Na}_{2}$ molecule after applying the pulses obtained using the three different optimization schemes: the momentum-based target ("vel"), the ionization target with fixed nuclei ("static"), and with moving nuclei ("dynamic"). We also plot the ionization obtained with the laser tuned to the resonance frequency pulse ("resonant"). Right panels (b, d): separation between the nuclei from their equilibrium position. Top panels (a) and (b) correspond to 16-fs pulses; bottom panels (c) and (d) correspond to 64-fs pulses.

(3) Using the same target as in case 2, but letting the nuclei move freely during the optimization, i.e., using OCT on top of the full EMD-TDDFT model.

Once we obtained the optimal pulses, we checked their performance by doing time propagations with them for much longer times. We also used larger simulation boxes for these test runs (which are the ones displayed below) to make sure that the results were fully converged with respect to the box sizes (given that ionization is the process that we are interested in) and to allow the nuclei to travel far without reaching the simulation box boundaries. Finally, we also performed equal test runs, making use of a quasimonochromatic laser pulse defined as the one in Eq. (22), but tuned in each case to the most relevant excitation frequency of the system. The goal was to check how the optimal pulses do a better job at Coulombexploding the cluster than simple pulses tuned to make use of the resonant-enhanced ionization phenomenon.

\section{A. $\mathrm{Na}_{2}$}

The bond length of the $\mathrm{Na}_{2}$ molecule is 5.82 a.u. [51], but for the simulations we have used the bond length computed by OCTOPUS within the LDA approximation: 5.48 a.u., an underestimation which is to be expected for the LDA. The polarization of the laser field was chosen to be parallel to the dimer axis.

Figure 1 shows the ionization induced in the molecule and the separation between the nuclei obtained from the pulses found with each kind of the three optimization schemes described above, and with a pulse in resonance with the main excitation $(\omega=2.1 \mathrm{eV})$ at the chosen polarization direction. The top panels correspond to pulses with durations of $6 \tau_{0} \approx 16 \mathrm{fs}$, whereas the bottom panels are longer ones 


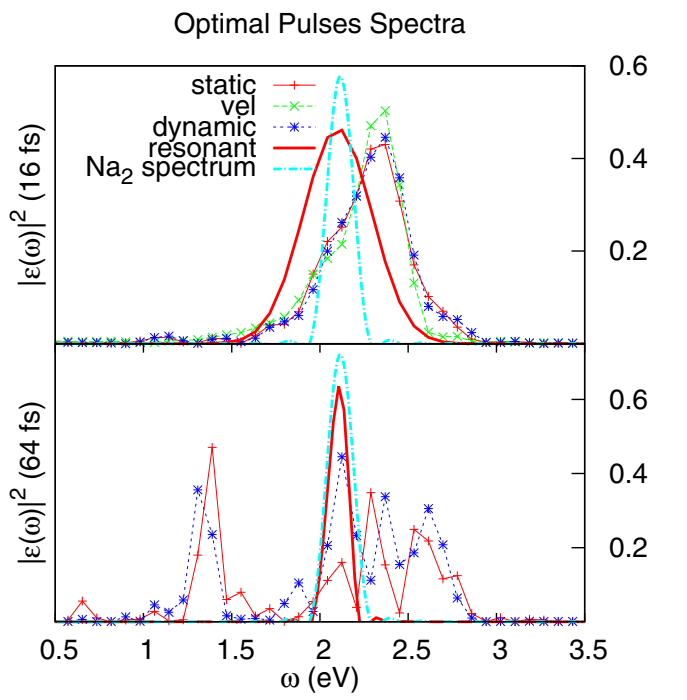

FIG. 2. Spectra of the optimized pulses and of the resonant pulse, and absorption spectrum of the $\mathrm{Na}_{2}$ molecule for light polarized along its axis. Top panel for the 16-fs pulses; bottom panel for the 64-fs pulses.

$\left(24 \tau_{0} \approx 64 \mathrm{fs}\right)$. For the former, we set the peak intensity of the reference pulse to $10^{12} \mathrm{~W} \mathrm{~cm}^{-2}$, and a lower intensity was used for the longer pulses $\left(3 \times 10^{11} \mathrm{~W} \mathrm{~cm}^{-2}\right)$.

As one can see, for the shorter pulses we have obtained Coulomb explosion for all three optimization schemes. The frequency spectra of these pulses and the absorption spectrum of the $\mathrm{Na}_{2}$ molecule (computed with the linear response formalism of TDDFT, within the adiabatic LDA) are plotted in Fig. 2 (top panel for the short pulses). All the solutions found are centered around the same frequency, slightly higher than the $2.1 \mathrm{eV}$ of the first resonance of the molecule. This blueshift of the optimal frequency should be blamed on ionization that is significant already within the pulse duration. In contrast, the nuclei do not move enough during the action of these short 16-fs pulses to produce a significant effect. This can be learned from the fact that the optimization performed with static nuclei provided a similar solution, both in terms of the ionic movement and the shape of the optimal pulses, than the two optimizations that do allow for ionic movement. Finally, it can be seen how a laser tuned to the ground-state resonance does not produce the photodissociation.

It is therefore clear that for those short pulses the ionic movement is not very relevant, which is in line with previous research on the Coulomb explosion of this kind of system [44]. We therefore tried longer pulses $\left(24 \tau_{0} \approx 64 \mathrm{fs}\right)$; the results are shown in the lower panels of Figs. 1 and 2. We used the "static" and "dynamic" optimization targets, and it can be seen how while the former was not capable of finding a dissociating pulse, the latter could. In other words, the ionic movement was sufficiently relevant to make the clamped nuclei approximation unsuitable, and therefore the full OCT+EMD+TDDFT combination was necessary. This can also be seen in Fig. 2, which shows how the optimal pulses obtained with the static and dynamic optimization schemes are significantly different.

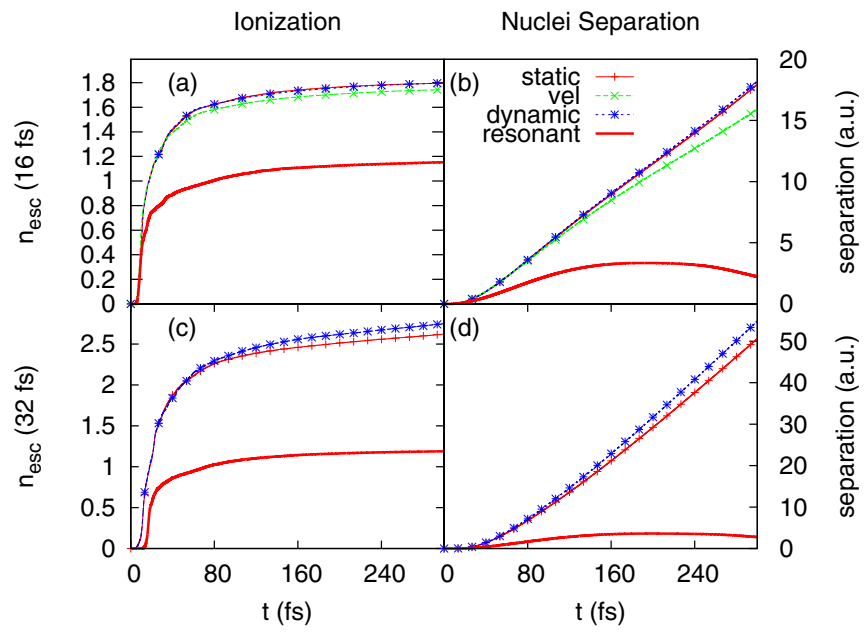

FIG. 3. Left panels (a, c): Electrons escaped from the $\mathrm{Na}_{4}$ cluster after applying the pulses obtained using the three different optimization schemes: the momentum-based target ("vel"), the ionization target with fixed nuclei ("static"), and with moving nuclei ("dynamic"). We also plot the ionization obtained with the laser tuned to the resonance frequency pulse ("resonant"). Right panels (b, d): Overall nuclear separation [Eq. (25)]. Top panels (a) and (b) correspond to 16-fs pulses; bottom panels (c) and (d) correspond to 32-fs pulses.

\section{B. $\mathrm{Na}_{4}$}

The lowest energy $\mathrm{Na}_{4}$ isomer is planar, with the four atoms forming a rhombus. We have attempted optimizations setting the polarization axis to coincide with both the short axis $(X)$ and the long axis $(Y)$ of the rhombus. We describe in detail only the former; for that direction, the main resonance of the system is at $\omega_{X}=2.7 \mathrm{eV}$. (For light polarized along the $Y$ axis, the resonance is found at $\omega_{Y}=1.9 \mathrm{eV}$.)

Figure 3 shows the ionization and the overall nuclear separation, defined as

$$
R(t)=\sum_{i<j}\left|\vec{R}_{i}(t)-\vec{R}_{j}(t)\right|-R_{\mathrm{eq}}
$$

where $R_{\text {eq }}$ is the value of the previous sum at time zero (equilibrium positions), so that $R(0)=0$. The results plotted correspond to calculations with the laser polarization along the $X$ axis. Figure 4 shows the power spectrum of the corresponding optimal pulses, along with the absorption spectrum of the cluster for light polarized in the $X$ direction.

We did calculations with 16-fs and 32-fs pulses in order to assess the relevance of the ionic motion. All optimization schemes produced optimal shapes capable of Coulombexploding the cluster, both with the shorter and with the longer pulses. For the shorter ones, the behavior of the system and the shape of the optimal pulse are very similar with all optimization schemes, including the static method in which the ionic movement is neglected. The results start to be different with 32-fs pulses: although both the static and dynamic schemes are successful, the shape of the optimal pulses (lower panel of Fig. 4) are different, which shows that the ionic movement starts to play a role. In all cases (short and long pulses, and with all optimization schemes), the main peaks of the frequency 


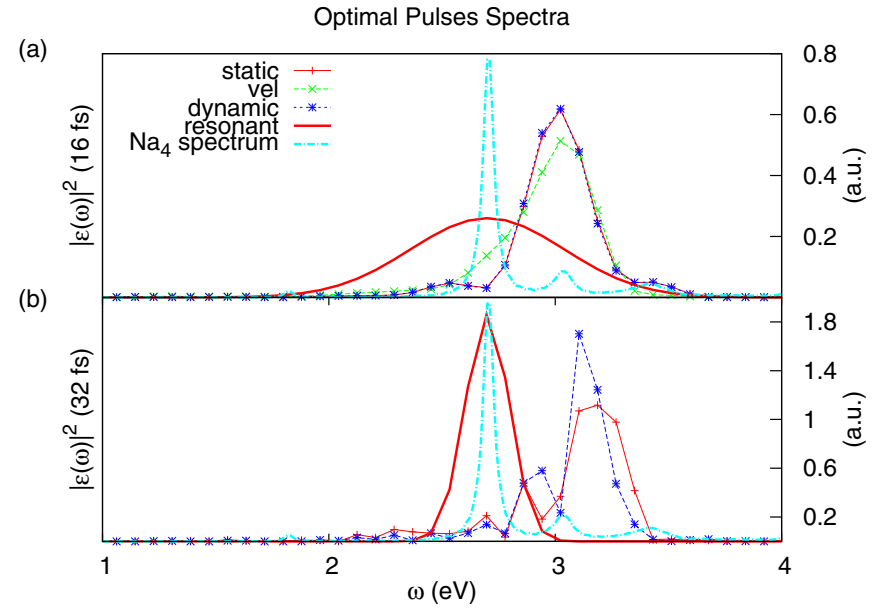

FIG. 4. Spectra of the optimized pulses and of the resonant pulse, and absorption spectrum of the $\mathrm{Na}_{4}$ molecule for light polarized along the $X$ axis. Top panel for the 16-fs pulses; bottom panel for the 32-fs pulses.

distributions are once again not over the resonance frequencies for the $X$ axis but are slightly blueshifted.

\section{C. $\mathrm{Na}_{8}$}

Finally, we show results for the $\mathrm{Na}_{8}$ cluster. The equilibrium geometry of this cluster has been the subject of some controversy [53], with various candidates with $D_{2 d}, D_{2 h}$, or $D_{4 d}$ symmetries. We have opted for the $D_{4 d}$ symmetry. This configuration is associated with a strong absorption peak (a plasmon resonance) at $2.55 \mathrm{eV}$ [53]. We set the polarization direction of the laser along the $Z$ axis.

In this case, we have only used 16-fs pulses due to the long computational times needed for this bigger system. Figure 5 shows the ionization of the cluster for both the "static" and "dynamic" ionization optimization schemes and for the pulse

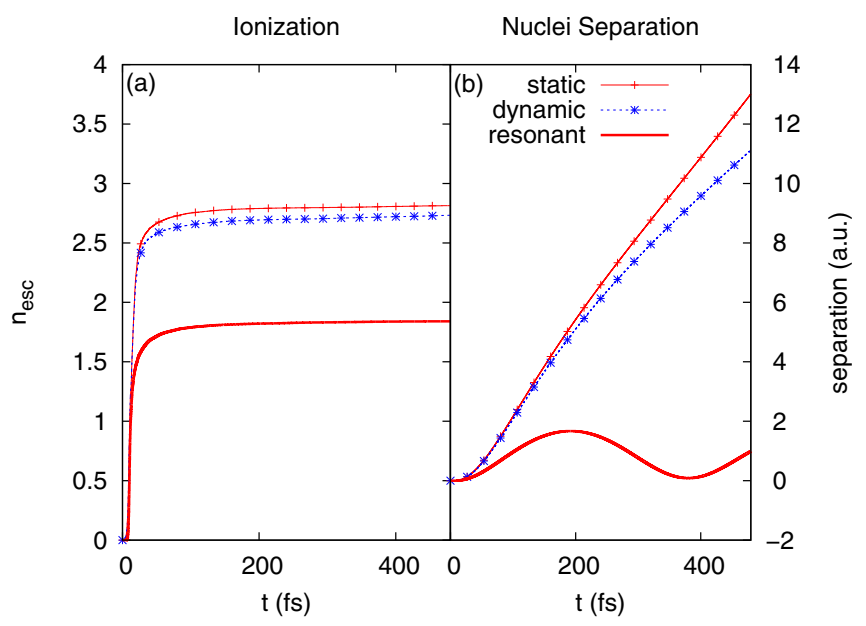

FIG. 5. (a) The ionization of the $\mathrm{Na}_{8}$ cluster after applying the pulses obtained using the static and dynamic nuclei ionization optimization schemes, using light polarized along the $Z$ axis. (b) The overall separation between the nuclei [Eq. (25)] that conform the $\mathrm{Na}_{8}$ cluster calculated with respect to the equilibrium separation.

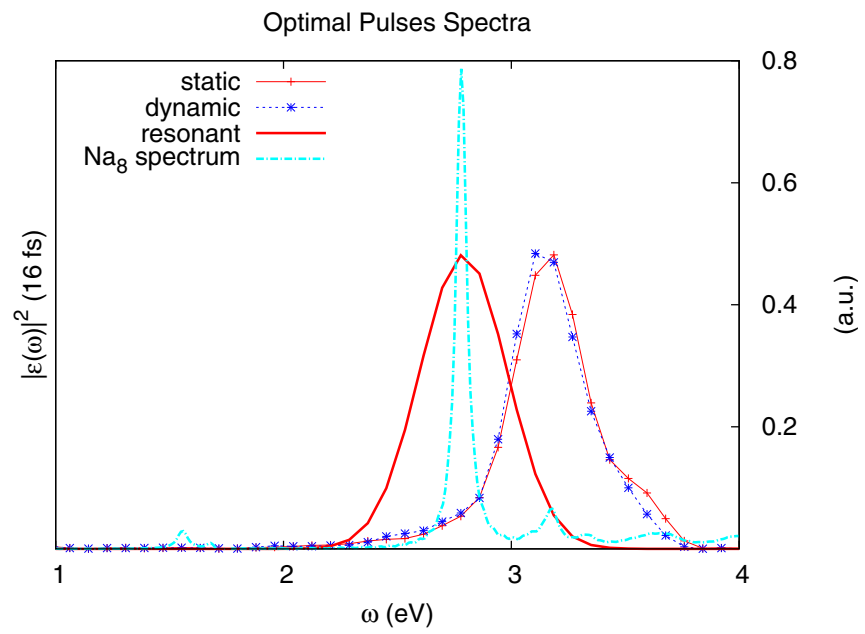

FIG. 6. Spectra of the optimized pulses and of the resonant pulses, and absorption spectrum of the $\mathrm{Na}_{8}$ cluster for light polarized along the $Z$ axis.

at the resonance frequency (which in this case is already a clear dominant plasmon peak). In the same figure, we show the overall separation between the nuclei that conform the cluster, as defined in Eq. (25). The use of both targets leads to successful solutions. Interestingly, the "static" nuclei optimization slightly improves the final ionization over the "dynamic" nuclei case. In both cases the ionization curve saturates at around 2.75, whereas the ionization obtained with the quasimonochromatic resonance frequency pulse lies below two electrons. In consequence, the nuclei oscillate only around their equilibrium position for this last case.

The two optimal pulses obtained with the different schemes have similar spectral composition, as can be seen in Fig. 6. Both are blueshifted with respect to the monochromatic resonant pulse. Once again, this shift must be attributed to the change in the plasmon resonance as the ionization takes place. Likewise, during the duration of the pulse, the ionic movement is small, which is manifest from the fact that both static and dynamic optimization schemes lead to similar results.

\section{CONCLUSIONS}

We have presented an implementation of OCT for the firstprinciples EMD-TDDFT model, which describes a system by considering classical point-particle nuclei and quantum electrons handled with TDDFT. We have described the details of its numerical implementation and have demonstrated its performance by optimizing the Coulomb explosion of small sodium clusters: the algorithm was set to find the optimal laser pulses capable of disintegrating the clusters for a given total duration, fluence, and cutoff frequency.

In order to find those optimal pulses, we have used a standard gradient-based nonlinear function maximization scheme for a merit function that values the suitability of a given pulse. In order to compute the gradient, the scheme needs consecutive forwards and backwards time propagations of the EMD-TDDFT equations, and of some related, but more difficult equations of motion for a fictitious auxiliary quantum-classical system. The main numerical difficulty lies 
precisely within these propagations: (1) the need of accurate enough gradients implies the need of a robust, yet expensive propagator such as fourth-order Runge-Kutta; (2) as we use absorbing boundaries in order to model the electron escape from the cluster, in the backwards propagation the norm of the KS orbitals increases and the propagation becomes unstable; (3) the equations for the auxiliary fictitious system (the "costate") contain a term that scales badly with the number of electrons (similarly to the exchange term in time-dependent Hartree-Fock).

We have chosen some small $\mathrm{Na}$ clusters to show the functionality of the theory and demonstrate how it can be used on larger and more complicated systems than the hydrogen two-electron systems that were used before as a proof of principle [40]. The process optimized was the Coulomb explosion of these clusters, a choice motivated by the interesting interplay between laser pulse, electron ionization, and ionic movement that was found in the past for these systems. It is to be expected that the explosion can be helped through the resonance-enhanced ionization phenomenon, i.e., by tuning the laser frequency to the plasmon resonance of the cluster. However, as the laser ionizes the clusters, the main resonance of these cluster blueshifts, and as the ions start to separate from each other, the same resonance redshifts. It is not clear a priori what frequency to use-or what frequencies, for a pulse with complex structure.

It is therefore a problem suitable for an OCT calculation, as the optimal solution cannot simply be guessed by intuition. We defined two optimization targets: the opposing momenta between pairs of atoms at the end of the pulse and the number of escaped electrons - the latter studied with both static and dynamic ions. The scheme proved successful and we observed the following: (1) The nuclei movement, for short 16-fs pulses, is not relevant, and the optimal solutions can be found ignoring it, i.e., using OCT on top of electronic-only TDDFT. (2) For longer 32-fs pulses, some differences between the optimal pulses obtained with and without ionic movement start to appear, as shown in the calculations for the $\mathrm{Na}_{4}$ cluster. (3) For even longer 64-fs pulses, the differences are substantial, and the OCT that ignores the ionic movement may even not be capable of finding a successful shape, making the full OCT on top of the nonadiabatic MD necessary. (4) The escape of electrons during the action of the pulse, even for the shortest 16-fs ones, is sufficiently large to shift the resonances, and therefore the optimal solutions do not have their main peak at ground-state resonances (as one would expect from a simple intuition based on the resonantly enhanced ionization idea) but at slightly blueshifted values.

\section{ACKNOWLEDGMENT}

We acknowledge support from the Ministerio de Economía y Competitividad (MINECO) through Grants No. FIS201346159-C3-2-P and No. FIS2014-61301-EXP.

\section{APPENDIX: COMPUTATIONAL ASPECTS}

\section{Optimization algorithms}

The problem of finding the maximum of a function depending on many variables is one of the most important in numerical analysis. Not surprisingly, there are a plethora of available methods, and their suitability will, of course, depend on factors such as the shape of the function (i.e., is it continuous? is it differentiable?), the number of parameters, etc. In the field of QOCT, several "ad hoc" algorithms were soon proposed [54-58] and we have implemented a number of those in OCTOPUS. However, many of these lack generality: for example, they may assume a particular parametrization of the control function (in our case, the electric amplitude $\varepsilon(t)$ ), such as the full time-discretization of the function in the time axis (which can be understood as not parameterizing the control function at all). This fact makes it sometimes difficult to impose constraints on the shape of the functions, etc. Moreover, they are designed for pure quantum OCT, and not suitable for the combined quantum and classical scheme discussed here.

Therefore, we have preferred to rely on general-purpose optimization algorithms, such as the low-storage BFGS algorithm $[49,50]$.

\section{Fourth-order accuracy in the gradient calculation}

The function maximization will only proceed successfully with any algorithm if the gradient is computed with sufficient accuracy. This is a challenging issue for the present problem, since the gradient computation requires a complex and long numerical procedure-the propagation of the differential equations shown above, followed by the integration in Eq. (12). The key numerical parameter is the time discretization step $\Delta t$. In our experience, we found it necessary to perform all operations with $\Delta t^{4}$ order accuracy. Otherwise, the error escalates fast with increasing total propagation time $T$. This implies time propagation algorithms of that order (to be explained in the next point) and an integration of Eq. (12) with a suitable order four scheme, such as Simpson's rule, i.e., for $t_{j}=\Delta t j,(j=0,1, \ldots, N)$ :

$$
\begin{aligned}
\int_{0}^{T} d t y(t)= & \frac{\Delta t}{3}\left[y(0)+2 \sum_{j=1}^{N / 2-1} y\left(t_{2 j}\right)\right. \\
& \left.+4 \sum_{j=1}^{N / 2} y\left(t_{2 j-1}\right)+y(T)\right] .
\end{aligned}
$$

With these precautions, the gradient computed with a time step $\Delta t, \vec{\nabla} G_{\Delta t}$ will differ from the exact one $\vec{\nabla} G_{0}$ by a fourthorder error in the time step:

$$
\left|\vec{\nabla} G_{\Delta t}-\vec{\nabla} G_{0}\right| \sim O\left(\Delta t^{4}\right) .
$$

\section{Propagation scheme}

We have implemented various propagation schemes for the TDKS equations in OCTOPUS [59]. As mentioned above, a fourth-order integrator is required for these calculations. Note that one must not only propagate the KS system of electrons, but the full mixed quantum and classical system determined by the full set of variables for the real system state. We may denote by $Y=(R, P, \varphi)$ to this full set of variables, where $R$ and $P$ are all the classical position and momenta variables, and $\varphi$ are the KS orbitals. For the costate, $Y=(\tilde{R}, \tilde{P}, \chi)$. Generically speaking, we face the propagation of first-order 
nonlinear differential equations in the form

$$
\dot{Y}=f[Y(t), t],
$$

where the dynamical function $f$ is given by the equations of motion above, different for state and costate. A fourth-order accurate, all-purpose time propagator is the classical explicit Runge-Kutta scheme (RK4):

$$
\begin{gathered}
Y(t+\Delta t)=Y(t)+\frac{\Delta t}{6}\left(K_{1}+2 K_{2}+2 K_{3}+K_{4}\right) \\
K_{1}=f(Y(t), t) \\
K_{2}=f\left(Y(t)+\Delta t / 2 K_{1}, t+\Delta t / 2\right) \\
K_{3}=f\left(Y(t)+\Delta t / 2 K_{2}, t+\Delta t / 2\right) \\
K_{4}=f\left(Y(t)+\Delta t K_{3}, t+\Delta t\right)
\end{gathered}
$$

Each time step, therefore, requires four evaluations of $f$. For the state, this implies the generation of the KS Hamiltonian for the given nuclear configuration and electronic density, its application on the set of KS orbitals, and the computation of the force, given in Eq. (7). The costate propagation is more complicated. First of all, note that the equation for the costate orbitals $\chi_{m}$ [Eq. (16)] contains two extra terms, in addition to the KS Hamiltonian: the second term in the right-hand side consists of the application of the operators $\hat{K}_{m n}[\varphi(t)]$. These are nonlocal operators, similar to the nonlocal operators used in Hartree-Fock theory. One needs to apply one of these operators for each Kohn-Sham orbital, and this fact makes the costate propagation to scale badly with the system size (roughly fourth order with the number of electrons), in contrast to the KS orbital propagation, which in principle is only second order. Moreover, the $\hat{K}_{m n}[\varphi(t)]$ operators are not Hermitian due to the presence of the imaginary part in their definition. Finally, the last term in the right-hand side makes the equation inhomogeneous.

The "real" state $(R, P, \varphi)$ could be propagated by making use of any of various methods suitable to propagate KohnSham equations (such as the ones described in Ref. [59]), in combination with, for example, the standard velocity-Verlet algorithm for the classical ions. However, due to the extra difficulties mentioned above, we have found that for the costate $(\tilde{R}, \tilde{P}, \chi)$ the robust all-purpose explicit RK4 scheme is the best option, despite its extra computational cost.

\section{Non-Hermitian evolution}

The equation of motion for the $\chi_{m}$ orbitals contains some non-Hermitian operators. This implies that the equations do not preserve the orbital norms - also because of the presence of one inhomogeneous term. For the purpose of the optimization shown below, moreover, we add a non-Hermitian term to the KS Hamiltonian of Eq. (6): an absorbing boundary potential that is used to account, in an approximate manner, for the possibility of ionization. The idea is to split the simulation region into an inner region in which the absorbing potential is zero and an outer region in which the action of the potential, due to its imaginary value, removes electron charge. In our calculations, the absorbing region has width $L$ and the absorbing potential definition is given by

$$
V_{\mathrm{abs}}(\vec{r})=i \eta \sin ^{2}\left(\frac{d(\vec{r}) \pi}{2 L}\right),
$$

where $d(\vec{r})$ is the distance from point to $\vec{r}$ to the frontier between inner and outer simulation box regions. The integral of the electron density in the simulation region,

$$
N(t)=\int_{V} d^{3} r n(\vec{r}, t),
$$

is no longer a constant of motion, and the electron loss may serve to estimate the ionization probability of the process.

Note that the propagation of the costate requires the prior knowledge of the true state evolution. One could perform the forward propagation of the state and store it at all times. This storage should be on disk because of its enormous size. The input and output operations required are too slow, and therefore the best solution is actually to recompute the system state at all times by propagating it backwards along with the costate.

When marching forwards, the norm of the KS orbitals decreases due to the presence of the absorbing boundaries and this makes the propagation a stable numerical procedure (since the error is proportional to the norm). However, when marching backwards, the norm increases, and the evolution is unstable. This can only be cured by establishing some milestone points during the forward propagation at which the orbitals are stored: the orbitals propagated backwards are compared to the orbitals stored at those points, and if they differ by an unacceptable amount, the program stops with an error; otherwise, the backwards-propagated orbitals are substituted by the stored ones.

\section{Operator derivatives}

The equations of motion contain various terms of the form

$$
\vec{\nabla}_{\mathbf{R}_{a}} f\left(\hat{\vec{r}}-\vec{R}_{a}\right)
$$

i.e., derivatives of operators that depend on the ionic positions. Numerically, in our real-space approach used by the OCTOPUS code, it is advantageous to use for their computation the identity:

$$
\vec{\nabla}_{\vec{R}_{a}} f\left(\hat{\vec{r}}-\vec{R}_{a}\right)=-i\left[\hat{\vec{p}}, f\left(\hat{\vec{r}}-\vec{R}_{a}\right)\right] .
$$

This permits us to substitute the numerical derivatives of the function $f$ by numerical derivatives of the wave functions (see Ref. [60] for a discussion on these issues). However, Eq. (15) also contains the derivatives of the forces

$$
\vec{\nabla}_{\alpha} \sum_{\beta} \tilde{\vec{R}}_{\beta}(t) \cdot \vec{F}_{\beta}[R(t), \varphi(t), u, t],
$$

which are, in fact, double derivatives with respect to the potential function. For these terms, we have employed a finite-difference scheme, i.e., the derivatives are estimated by computing the forces at neighboring values of the nuclear positions.

\section{Control function parametrizations}

We allow for various possible parametrizations $\varepsilon=$ $\varepsilon\left(u_{1}, \ldots, u_{M}, t\right)$ in our optimal control implementation in the 
OCTOPUS code. One simple possibility is using directly the real-time discretization

$$
u_{j}=\varepsilon\left(t_{j}\right)
$$

However, this implies a large number of parameters. Moreover, it is hard to establish constraints on the function, such as a cutoff frequency, for example. For the examples shown below, we have used the parametrization described in Ref. [61], based on a Fourier expansion:

$$
\varepsilon(u, t)=\sum_{n=0}^{K}\left[a_{n}(u) \cos \left(\frac{2 \pi}{T} n t\right)+b_{n}(u) \sin \left(\frac{2 \pi}{T} n t\right)\right] .
$$

This form naturally sets a cutoff frequency on the shape of the control function, $\omega_{\max }=\frac{2 \pi}{T} K$. The parameters $u$ are a set of hyperspherical angles that run over all possible Fourier coefficients $a_{n}, b_{n}$, subject to the following constraints:

$$
\begin{gathered}
\varepsilon(u, 0)=\varepsilon(u, T)=0, \\
\int_{0}^{T} d t \varepsilon(u, t)=0, \\
\int_{0}^{T} d t \varepsilon^{2}(u, t)=F_{0} .
\end{gathered}
$$

The first two conditions are natural restrictions for an electric field produced by a laser pulse. The last condition establishes a "fixed-fluence" condition: the search is done over all possible laser pulses whose intensity integrated in time (the fluence) is given. A condition over the energy of the pulse such as this one is necessary for our optimization target, since otherwise the obvious solution to Coulomb-explode a cluster would be a pulse with infinite intensity.
[1] U. Keller, Nature (London) 424, 831 (2003).

[2] A. H. Zewail, Femtochemistry: Ultrafast Dynamics of the Chemical Bond (World Scientific Publishing, Singapore, 1994), Vols. I \& II.

[3] F. Krausz and M. Ivanov, Rev. Mod. Phys. 81, 163 (2009).

[4] D. C. Rapaport, The Art of Molecular Dynamics Simulation (Cambridge University Press, Bar-Ilan University, Israel, 2004).

[5] G. Stefanucci and R. van Leeuwen, Nonequilibrium Many-Body Theory of Quantum Systems (Cambridge University Press, Cambridge, 2013).

[6] E. Runge and E. K. U. Gross, Phys. Rev. Lett. 52, 997 (1984).

[7] Fundamentals of Time-Dependent Density Functional Theory, edited by M. A. Marques, N. T. Maitra, F. M. Nogueira, E. Gross, and A. Rubio (Springer, Berlin, 2012).

[8] R. B. Gerber, V. Buch, and M. A. Ratner, J. Chem. Phys. 77, 3022 (1982).

[9] G. Wentzel, Eur. Phys. J. A 38, 518 (1926).

[10] H. A. Kramers, Eur. Phys. J. A 39, 828 (1926).

[11] L. Brillouin, C. R. Acad. Sci. Paris 183, 24 (1926).

[12] F. A. Bornemann, P. Nettesheim, and C. Schütte, J. Chem. Phys. 105, 1074 (1996).

[13] J. Theilhaber, Phys. Rev. B 46, 12990 (1992).

[14] U. Saalmann and R. Schmidt, Z. Phys. D: At., Mol. Clusters 38, 153 (1996).

[15] F. Calvayrac, P.-G. Reinhard, and E. Suraud, J. Phys. B: At., Mol. Opt. Phys. 31, 5023 (1998).

[16] T. Kunert and R. Schmidt, Phys. Rev. Lett. 86, 5258 (2001).

[17] A. Castro, M. A. L. Marques, J. A. Alonso, G. F. Bertsch, and A. Rubio, Eur. Phys. J. D 28, 211 (2004).

[18] Y. Miyamoto and H. Zhang, Phys. Rev. B 77, 045433 (2008).

[19] A. Russakoff, S. Bubin, X. Xie, S. Erattupuzha, M. Kitzler, and K. Varga, Phys. Rev. A 91, 023422 (2015).

[20] A. H. Zewail, Phys. Today 33, 27 (1980).

[21] P. Brumer and M. Shapiro, Chem. Phys. Lett. 126, 541 (1986).

[22] C. K. Chan, P. Brumer, and M. Shapiro, J. Chem. Phys. 94, 2688 (1991).

[23] C. Chen, Y.-Y. Yin, and D. S. Elliott, Phys. Rev. Lett. 64, 507 (1990).
[24] D. J. Tannor and S. A. Rice, J. Chem. Phys. 83, 5013 (1985).

[25] U. Gaubatz, P. Rudecki, M. Becker, S. Schiemann, M. Külz, and K. Bergmann, Chem. Phys. Lett. 149, 463 (1988).

[26] R. S. Judson and H. Rabitz, Phys. Rev. Lett. 68, 1500 (1992).

[27] A. Assion, T. Baumert, M. Bergt, T. Brixner, B. Kiefer, V. Seyfried, M. Strehle, and G. Gerber, Science 282, 919 (1998).

[28] S. A. Rice and M. Zhao, Optical Control of Molecular Dynamics (Wiley, New York, 2000).

[29] M. Shapiro and P. Brumer, Quantum Control of Molecular Processes, 2nd ed. (Wiley-VCH Verlag GmbH \& Co. KGaA, New York, 2012).

[30] C. Brif, R. Chakrabarti, and H. Rabitz, New J. Phys. 12, 075008 (2010)

[31] S. Shi, A. Woody, and H. Rabitz, J. Chem. Phys. 88, 6870 (1988).

[32] A. P. Peirce, M. A. Dahleh, and H. Rabitz, Phys. Rev. A 37, 4950 (1988).

[33] R. Kosloff, S. Rice, P. Gaspard, S. Tersigni, and D. Tannor, Chem. Phys. 139, 201 (1989).

[34] W. Jakubetz, J. Manz, and H.-J. Schreier, Chem. Phys. Lett. 165, 100 (1990).

[35] J. Werschnik and E. K. U. Gross, J. Phys. B: At., Mol. Opt. Phys. 40, R175 (2007).

[36] A. Castro, J. Werschnik, and E. K. U. Gross, Phys. Rev. Lett. 109, 153603 (2012).

[37] C. D. Schwieters and H. Rabitz, Phys. Rev. A 48, 2549 (1993).

[38] J. Botina, H. Rabitz, and N. Rahman, J. Chem. Phys. 102, 226 (1995).

[39] A. Castro and E. K. U. Gross, J. Phys. A: Math. Theor. 47, 025204 (2013).

[40] A. Castro, Chem. Phys. Chem. 17, 1601 (2016).

[41] T. Fennel, K.-H. Meiwes-Broer, J. Tiggesbäumker, P.-G. Reinhard, P. M. Dinh, and E. Suraud, Rev. Mod. Phys. 82, 1793 (2010).

[42] F. Calvayrac, P.-G. Reinhard, E. Suraud, and C. Ullrich, Phys. Rep. 337, 493 (2000).

[43] P. Wopperer, P. Dinh, P.-G. Reinhard, and E. Suraud, Phys. Rep. 562, 1 (2015). 
[44] E. Suraud and P. G. Reinhard, Phys. Rev. Lett. 85, 2296 (2000).

[45] L. Ma, E. Suraud, and P.-G. Reinhard, Eur. Phys. J. D 14, 217 (2001).

[46] P. Schwerdtfeger, Chem. Phys. Chem. 12, 3143 (2011).

[47] M. Marques, Comput. Phys. Commun. 151, 60 (2003).

[48] A. Castro, H. Appel, M. Oliveira, C. A. Rozzi, X. Andrade, F. Lorenzen, M. A. L. Marques, E. K. U. Gross, and A. Rubio, Phys. Status Solidi B 243, 2465 (2006).

[49] J. Nocedal, Math. Comput. 35, 773 (1980).

[50] D. C. Liu and J. Nocedal, Math. Program. 45, 503 (1989).

[51] R. D. J. III, NIST computational chemistry comparison and benchmark database, NIST standard reference database number 101 (2015).

[52] I. A. Solov'yov, A. V. Solov'yov, and W. Greiner, Phys. Rev. A 65, 053203 (2002).
[53] G. Pal, G. Lefkidis, H. C. Schneider, and W. Hübner, J. Chem. Phys. 133, 154309 (2010).

[54] W. Zhu, J. Botina, and H. Rabitz, J. Chem. Phys. 108, 1953 (1998).

[55] W. Zhu and H. Rabitz, J. Chem. Phys. 109, 385 (1998).

[56] D. J. Tannor, V. Kazakov, and V. Orlov, in Nato ASI Series (Springer Science + Business Media, New York, 1992), pp. 347-360.

[57] J. Somlói, V. A. Kazakov, and D. J. Tannor, Chem. Phys. 172, 85 (1993).

[58] Y. Maday and G. Turinici, J. Chem. Phys. 118, 8191 (2003).

[59] A. Castro, M. A. L. Marques, and A. Rubio, J. Chem. Phys. 121, 3425 (2004).

[60] K. Hirose, T. Ono, Y. Fujimoto, and S. Tsukamoto, FirstPrinciples Calculations in Real-Space Formalism (World Scientific Publishing, Singapore, 2005).

[61] K. Krieger, A. Castro, and E. Gross, Chem. Phys. 391, 50 (2011). 